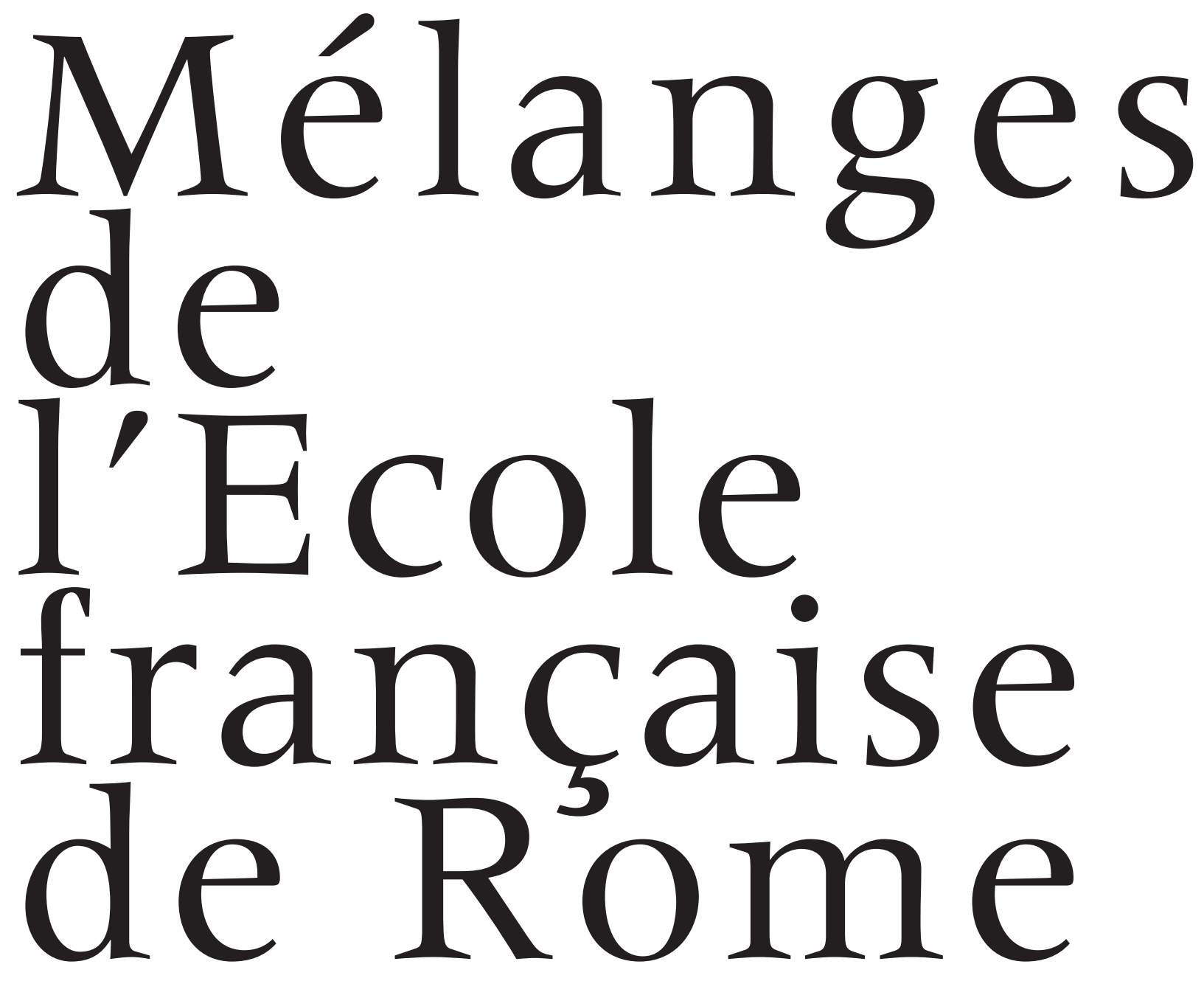

Antiquité

MEFRA

131-2 2019 


\title{
Le copie ad acquerello delle pitture funerarie etrusche nel Museo delle Antichità Etrusche e Italiche della Sapienza Università di Roma
}

\author{
Claudia Carlucci e Laura M. Michetti
}

Cl. Carlucci, Sapienza Università di Roma, Polo Museale Sapienza, claudia.carlucci@uniromal.it

L.M. Michetti, Sapienza Università di Roma, Dipartimento di Scienze dell'Antichità, laura.michetti@uniromal.it

Le copie delle pitture funerarie etrusche conservate nel Museo delle Antichità Etrusche e Italiche della Sapienza Università di Roma furono acquistate negli anni Cinquanta del secolo scorso da Massimo Pallottino presso Francesca Fioroni Barlanti, nuora del primo proprietario, Vincenzo Fioroni. Sono presenti nel patrimonio del museo universitario fin dal momento della sua fondazione nel 1955. La redazione delle 61 tavole - relative a 24 tombe di Tarquinia e alle tombe della Scimmia di Chiusi e Golini I di Orvieto - finora praticamente inedite, si colloca, per quanto riguarda la serie tarquiniese, negli anni 1911-1912, in seguito alla svolta decisiva rappresentata dall'utilizzo di riproduzioni fotografiche a supporto del lavoro dei copisti. Documenti d'archivio riportano il nome del pittore, l'altrimenti ignoto Elio D'Alessandris, e il suo rapporto, non precisato, con l'Accademia romana di Belle Arti. L'importanza della serie conservata presso il Museo della Sapienza risiede nel rappresentare il ciclo completo delle tombe dipinte conosciute a Tarquinia agli inizi del ‘900. Nulla sappiamo, invece, dell'autore delle copie delle tombe di Chiusi e Orvieto.

Pitture etrusche, Museo delle Antichità Etrusche e Italiche, Vincenzo Fioroni, Elio D’Alessandris

The watercolors copies of Etruscan tomb painting in the Museum of Etruscan and Italic Antiquities at the Sapienza University of Rome. The copies of the Etruscan tomb paintings preserved at the Museo delle Antichità Etrusche e Italiche, Sapienza University of Rome, were purchased in the 1950s by Massimo Pallottino from Francesca Fioroni Barlanti, daughter of the first owner, Vincenzo Fioroni. They have formed part of the collection of the university museum since its foundation in 1955. The 61 panels, which relate to the 24 tombs of Tarquinia, the Tomba della Scimmia of Chiusi and the Tomba Golini I of Orvieto, have remained unpublished to date. With regard to the Tarquinia series, it is believed that they were drafted between 1911 and 1912, based on the application of the newly developed technique of using photographic reproductions to support the work of copyists. The otherwise unknown Elio D'Alessandris is recorded as the painter of these works in the archive documents, as is his relationship with the Roman Academy of Fine Arts, although the nature of this relationship is not specified. The author of the copies of the tombs at Chiusi and Orvietto has, on the other hand, still not been identified. The importance of the Sapienza Museum series stems from the fact that it records the complete inventory of painted tombs known in Tarquinia at the beginning of the twentieth century.

Etruscan tomb paintings, Museo delle Antichità Etrusche e Italiche, Vincenzo Fioroni, Elio D’Alessandris 
Le copie ad acquerello delle pitture funerarie etrusche nel Museo delle Antichità Etrusche e Italiche

404 Claudia Carlucci e Laura Michetti

\section{VINCENZO FIORONI E LE COPIE AD ACQUERELLO}

Le copie ad acquerello di pitture funerarie etrusche della Sapienza sono uno dei segni di quella sorta di fervore etruscologico che si fa progressivamente più vivo nel corso della prima metà del Novecento. ${ }^{1}$

Le 61 tavole - finora praticamente inedite se si eccettuano alcune sporadiche riproduzioni di singole tombe ${ }^{2}$ - già collocate negli anni Cinquanta del secolo scorso nelle stanze dell'Istituto universitario diretto da Massimo Pallottino e presenti nel patrimonio del Museo delle Antichità Etrusche e Italiche fin dal momento della sua fondazione nel 1955, furono integralmente esposte a cura di Giovanni Colonna, allora assistente volontario della cattedra di Etruscologia e antichità italiche tenuta da Pallottino, in tre sale della Sezione artistica del Museo, il cui allestimento fu inaugurato il 29 agosto 1962 in occasione del VI Congresso Internazionale di Scienze Preistoriche e Protostoriche (fig. 1). ${ }^{3} \mathrm{Nel}$ 2000, in concomitanza con i lavori di ristrutturazione del Museo dell'Arte Classica sotto la direzione di Andrea Carandini, la maggior parte delle tavole

1. Ringraziando il Comitato scientifico che ci ha coinvolto in questa iniziativa, sentiamo la necessità di ricordare con affetto la nostra amica Luciana Drago. A lei, infatti, Giovanni Colonna affidò la pubblicazione degli acquerelli del Museo della Sapienza, opera che Luciana ha portato avanti con grandissimo impegno fino a pochi giorni prima della sua scomparsa. Il volume è attualmente nella serie dei Cataloghi del Museo. Il volume Il Museo delle Antichità Etrusche e Italiche della Sapienza Università di Roma, IV. Le copie delle pitture funerarie etrusche, era stato nel corso degli anni elaborato nelle sue linee generali da Luciana e organizzato in capitoli. Il recupero dei testi e la consulenza dei colleghi e collaboratori che l'hanno affiancata a diverso titolo nel tempo - in particolare Matteo Piccioni e Manuela Bonadies che ringraziamo per il supporto che ci hanno offerto - ci hanno consentito di procedere alla revisione complessiva del materiale nel rigoroso rispetto delle intenzioni dell'autrice e di approntare il lavoro per la stampa. Il presente contributo prende dunque le mosse dai principali dati emersi dalle ricerche di Luciana Drago, nell'ottica di richiamare sinteticamente l'attenzione su alcuni elementi che trovano nel volume uno sviluppo ben più esteso.

2. Cristofani 1971, p. 8, fig. 2-6, 12, 18, 20 (Tomba del Tifone); Morandi 1983, p. 27, fig. 26 (Tomba del Cardinale); Colonna 1984, p. $74-75$, fig. 1-4 (tombe dei Tori, dell'Orco II, delle Bighe, delle Leonesse); Drago Troccoli 2005, p. 17-19, fig. 10 (Tomba del Mare).

3. Sull'istituzione del museo e sulle sue collezioni, si veda in particolare Colonna 1993 e Drago Troccoli 2005, p. 7-17. Sulle copie della Sapienza, cenni in Lubtchansky 2017a, p. 33-34. è stata sistemata nella stessa Sezione - ora ridotta a tre sale, rispettivamente dedicate alla scultura e due alla pittura etrusca - inserita nel percorso di quel Museo, adiacente al settore scultura ellenistica. Un piccolo gruppo di tavole è stato invece inserito nell'allestimento del settore museale nel piano seminterrato della Facoltà di Lettere, a integrazione del materiale didattico esposto nelle sale dedicate all'architettura funeraria etrusca.

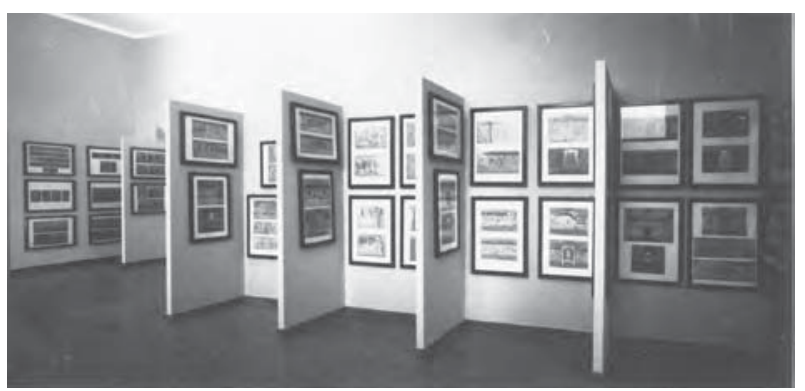

Fig. 1 - Le copie nell'allestimento del 1962 della Sezione artistica del Museo delle Antichità Etrusche e Italiche. (c) AMAEIS.

La redazione di questa serie di copie si colloca negli anni 1911-1912, in seguito quindi alla svolta decisiva rappresentata, nella seconda metà del XIX secolo, dall'utilizzo di riproduzioni fotografiche a supporto del lavoro dei copisti. Questa novità cambia infatti il modo di intendere le copie e la loro funzione: non più illustrazione delle diverse manifestazioni della pittura etrusca, per cui erano ammesse aggiunte o interpretazioni influenzate dalla cultura artistica prevalentemente classicistica dei copisti, ma documentazione fedele delle immagini leggibili sulle pareti delle tombe al momento della realizzazione delle copie. Anche le lacune e le integrazioni dovute rispettivamente ai guasti subiti nel tempo dopo l'apertura delle camere e ai relativi interventi di restauro vengono accuratamente riprodotte con l'ausilio in studio delle fotografie scattate sia dagli stessi pittori che da fotografi professionisti (fig. 2-3).

È il caso soprattutto di tre serie di copie, di livello decisamente diverso. La prima è quella conservata nell'Istituto svedese di Studi Classici a Roma, eseguita tra il 1897 e il 1908 dall'équipe di Alessandro Morani in 23 tombe di Tarquinia";

4. Su questa serie, vedi in particolare il recente catalogo della mostra, con bibliografia precedente, Capoferro - Renzetti 2017 e Capoferro in questo dossier. 
la seconda e la terza, confluite nel patrimonio del Museo delle Antichità etrusche e italiche della Sapienza, consistono nelle copie delle pitture delle tombe della Scimmia di Chiusi e Golini I di Orvieto - eseguite da un anonimo - e in quelle delle medesime 23 tombe integrate con la Tomba del Mare

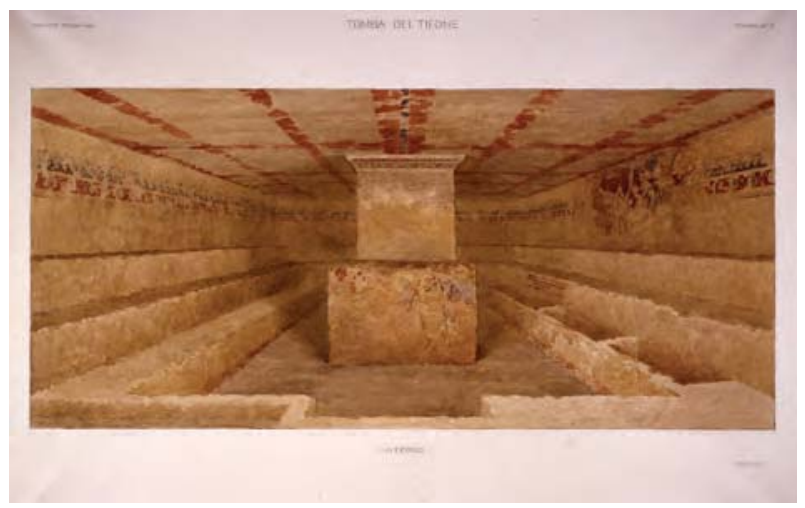

Fig. 2 - Acquerello, Tomba del Tifone, tomba n. X, tav. I. (C) AMAEIS.

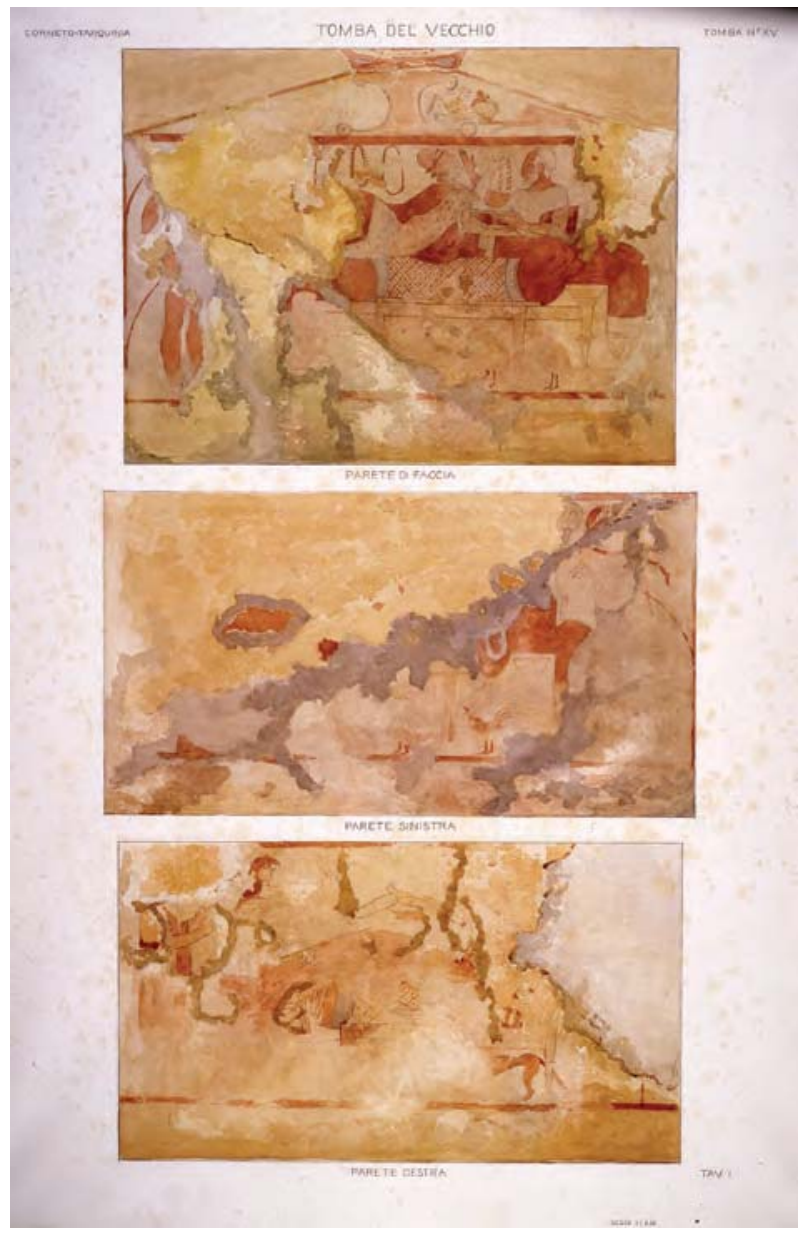

Fig. 3 - Acquerello, Tomba del Vecchio, tomba n. XV, tav. I. (c) AMAEIS. realizzate tra il 1911 e il 1912 dal pittore che operò esclusivamente a Tarquinia (fig. 4).

È probabile che non ci sia alcun rapporto tra questa più cospicua e omogenea serie di riproduzioni e le tavole delle tombe di Chiusi e Orvieto, per le quali non si dispone di alcun documento d'archivio: per queste ultime è quindi possibile solamente fare ipotesi basate sulle caratteristiche tecniche e stilistiche che le distinguono nettamente tra loro e dalle altre, attribuibili viceversa a un'iniziativa unitaria.

Del ciclo tarquiniese - il più completo dei decenni a cavallo tra XIX e XX secolo - ci restano solo le riproduzioni in scala ridotta che ne costituiscono l'esito finale. Queste permettono di apprezzare - al di là della scarsa precisione mostrata nei dettagli della decorazione pittorica dal pittore, il cui stile impressionistico rivela ancora un forte legame con il paesaggismo ottocentesco - l'efficacia e la fedeltà agli originali, limitatamente allo stato di conservazione, delle vedute d'insieme degli interni delle tombe, unita in molti casi a un intento realistico apprezzabile negli scorci del paesaggio esterno visto dai dromoi dei sepolcri. Si tratta insomma, più che di rielaborazioni di rilievi delle tombe ispirati

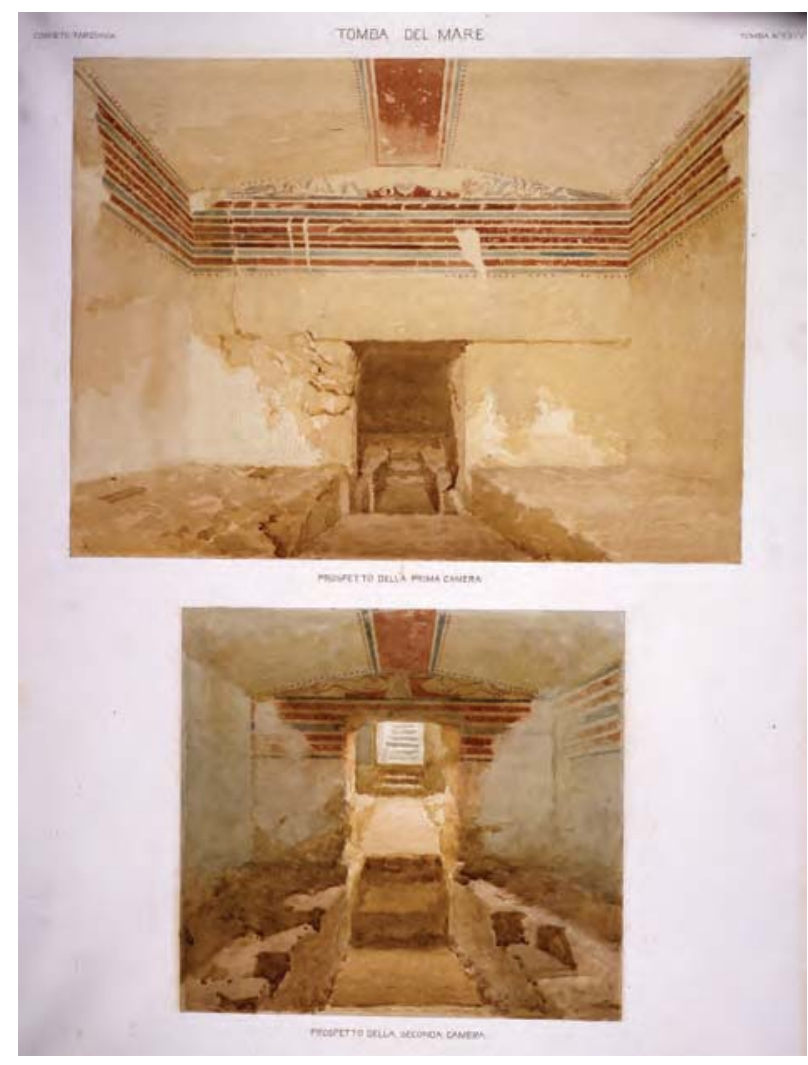

Fig. 4 - Acquerello, Tomba del Mare, tomba n. XXIV. (C) AMAEIS. 
Le copie ad acquerello delle pitture funerarie etrusche nel Museo delle Antichità Etrusche e Italiche

406 Claudia Carlucci e Laura Michetti

a un rigore scientifico, di vere e proprie finestre aperte sull'interno che ripropongono il punto di vista dell'ipotetico osservatore sulla scia delle inquadrature fotografiche che circolavano ampiamente in quegli anni.

L'acquisto è avvenuto all'inizio degli anni Cinquanta del secolo scorso, prima dell'istituzione del Museo, a seguito dell'offerta di vendita all'Università da parte di Francesca Fioroni Barlanti, nuora del primo proprietario, Vincenzo Fioroni. ${ }^{5}$ Le uniche notizie che si possono trarre dalla documentazione dell'Archivio del Museo, utili per l'attribuzione e l'inquadramento cronologico delle copie delle 24 tombe tarquiniesi accessibili nei primi decenni del Novecento, sono contenute in due brevi documenti.

Un biglietto manoscritto indirizzato a Pallottino in data 18 luglio 1967 da parte dell'amica di famiglia, Francesca Fioroni Barlanti, accompagna un foglio dattiloscritto contenente la seguente succinta nota biografica di Vincenzo Fioroni, compilata dal figlio Giuseppe su richiesta di Pallottino: ${ }^{6}$

Negli anni tra il 1905 e il 1914, Vincenzo Fioroni (nato nel 1867 morto nel 1929), intraprese scavi archeologici a Tarquinia nella zona "Monterozzi», dove furono rinvenute per la maggior parte tombe del tipo a pozzetto (scavate in massi di tufo).

Vennero alla luce urne cinerarie, elmi, spade, specchi, fibule e altro materiale minuto, misto ai frammenti delle ossa che, dopo la cremazione, venivano raccolte in un panno e collocate nelle urne cinerarie.

Il materiale rinvenuto si trova quasi tutto al Museo Etrusco di Firenze. Un frammento di sarcofago con una scritta etrusca si trova al Museo di Villa Giulia a Roma

Nello stesso periodo Vincenzo Fioroni, che aveva notato il progressivo deterioramento delle pitture che decoravano le pareti delle tombe etrusche di Tarquinia, incaricò il pittore Elio D'Alessandris dell'Accademia di Belle Arti di Roma, di eseguire le riproduzioni delle pitture stesse.

Portando la luce del sole nelle tombe, con un sistema di specchi, e con lampade a carburo, furono eseguite $\mathrm{N}^{\circ} 52$ tavole ad acquerello che sono la riproduzione in scala ridotta, ma identica in tutti i particolari di

5. Drago Troccoli 2005, p. 19.

6. I documenti sono conservati nell'Archivio del Museo delle Antichità Etrusche e Italiche della Sapienza. disegno e colore, delle pitture etrusche nelle condizioni in cui si trovavano circa 60 anni fa.

Sempre nello stesso periodo Vincenzo Fioroni fece eseguire, sotto la sua direzione, le copie dei più pregiati vasi etruschi e greci che si trovavano nei musei di Tarquinia e Firenze ${ }^{7}$.

Risulta particolarmente importante - in relazione alla fede da prestare alle notizie fornite dal figlio sulle attività di Vincenzo Fioroni (18671929) - la precisazione contenuta nella lettera della signora sulla partecipazione diretta del marito agli interessi e alle iniziative in campo archeologico del padre ${ }^{8}$, farmacista appartenente a una benestante famiglia romana, possessore di fondi a Tarquinia dove nei primi due decenni del Novecento è attivo con scavi dai quali trae discreti vantaggi economici. ${ }^{9}$ Interessanti le informazioni contenute nella nota biografica circa: 1) le motivazioni "ufficiali» che spinsero Fioroni ad affidare a un pittore le riproduzioni in scala ridotta delle tombe dipinte tarquiniesi allora note e accessibili, a seguito della constatazione del loro progressivo deterioramento (anche se è certa la finalità almeno in parte venale dell'operazione); 2) il periodo di realizzazione delle copie, in un anno imprecisato tra il 1905 e il 1914; 3) il nome del pittore, l'altrimenti ignoto Elio D'Alessandris, e il suo rapporto, invero non precisato, con l'Accademia romana di Belle Arti; 4) la tecnica d'illuminazione degli interni delle tombe, da questi utilizzata per realizzare le copie.

Decisamente più ricca è la documentazione conservata presso l'Archivio storico della ex Soprintendenza Archeologia della Toscana (sotto la cui giurisdizione si trovava Corneto-Tarquinia) che permette di datare più precisamente l'esecuzione delle copie tra il 1911 e il 1912, con un'interruzione forzata di quasi otto mesi tra la fine di settembre del 1911 e il 21 maggio del 1912, e di avere la conferma che il lavoro fu effettivamente eseguito all'interno delle tombe dal pittore

7. AMAEIS, memoria di Giuseppe Fioroni.

8. AMAEIS, lettera del 18.07.1967: "Caro Professore, le mando questa nota scritta da mio marito che da ragazzo seguiva suo padre nelle sue attività "archeologiche" ".

9. Fioroni risulta tra l'altro autore di un volume dedicato Alle arti di Klitias Ergotimos e Fintias. Copie dei migliori vasi greci antichi, per lo più di vasi etruschi e greci conservati nei musei di Tarquinia e Firenze, per eseguire le quali fece costruire appositamente una fabbrica nel giardino della sua casa tarquiniese. Colonna 1984, p. 75 
menzionato dal figlio di Fioroni, purtroppo mai nominato direttamente ma solo con gli epiteti «il pittore» $\mathrm{O}$ "l'artista» ${ }^{10}$.

Il primo documento attestante l'interesse di Fioroni a far riprodurre le pitture delle tombe tarquiniesi e le relative reazioni delle istituzioni coinvolte è la lettera che segue, indirizzata al Ministro della Pubblica Istruzione da Luigi Adriano Milani allora a capo della Regia Soprintendenza ai Musei e Scavi d'Etruria e direttore del Regio Museo Archeologico di Firenze:

Firenze 3 luglio 1911

\section{A S.E. il Ministro della P.I. Roma}

In relazione con la mia lettera 2 luglio corrente riguardante la costituenda Galleria delle pitture etrusche in fac-simile devo richiamare l'attenzione dell'E.V. sopra una concessione fatta dall'Ufficio dei Monumenti di Roma, ad insaputa di questa Soprintendenza, al Sig. ${ }^{r}$ Fioroni di Corneto, di copiare le pitture delle tombe della necropoli tarquiniese. Il custode dei monumenti di Corneto [Edmondo La Valle] costretto a presenziare giornalmente a tale lavoro di riproduzione dei dipinti delle tombe, perché non abbiano a commettersi guasti di sorta, ha inviato a questa Soprintendenza copia di una lettera che egli diresse all'Ufficio dei Monumenti di Roma, dal quale dipende, chiedendo un adeguato compenso per questo incarico straordinario. Mentre comunico siffatta lettera, devo rilevare dal mio canto che il Servizio di assistenza ai copiatori dei dipinti delle tombe, oltreché riuscire gravoso per il detto funzionario, lo distoglie dai suoi più diretti obblighi di ufficio che è la vigilanza ai monumenti e l'accompagnamento dei visitatori alle tombe. Tenuto conto che le concessioni per copie di dipinti delle tombe fatte da privati, come sarebbe il caso del Sig. ${ }^{r}$ Fioroni, hanno palesemente uno scopo commerciale o di speculazione industriale e che accanto a siffatte richieste non ne mancheranno delle altre da parte di concorrenti alla medesima speculazione essendovi anche in corso domande per parte della Gliptoteca Ny Carlsberg di Kopenhagen che vuol completare la sua collezione, io ritengo che non vi sia altro provvedimento che di sollecitare la copia campione fatta direttamente dallo stato presso il mu-

10. Così indicato in due lettere manoscritte, rispettivamente di E. La Valle al Milani del 7 luglio 1911, ASAT, prot. 1154, Oggetto: Tombe dipinte, e di Fioroni al Milani del 4 maggio 192, ASAT, pos. X/C, prot. 253. seo centrale della civiltà etrusca. Così l'inconveniente di tante domande sarebbe evitato con grande vantaggio degli originali soggetti a continui danni, e sarebbe evitato pure l'oneroso servizio di vigilanza, potendo e privati e musei esteri ritrarre le riproduzioni dalla detta copia campione. A tale proposito mi riferisco alla mia lettera 2 luglio sulla costituenda Galleria della pittura etrusca in fac-simile dove ho enumerato dettagliatamente tutti i vantaggi che deriverebbero con l'attuazione di così importante progetto. ${ }^{11}$

Dal testo, particolarmente interessante ai fini della ricostruzione del quadro storico-culturale degli anni che vedono la conclusione dell'impresa Helbig-Morani, emerge con chiarezza l'ostilità del Milani nei confronti di chiunque ostacolasse o si sovrapponesse al suo ambizioso e quasi ossessivo disegno di realizzare una Galleria della pittura etrusca in facsimile nel Museo di Firenze che superasse, piuttosto che emulare, qualsiasi iniziativa avviata in precedenza, in particolare quella di Carl Jacobsen, ${ }^{12}$ da Milani come da altri in quel tempo definito per spregio «il birraio», nella Ny Carlsberg Glyptotek di Copenaghen. ${ }^{13}$

L'ostilità nei confronti di Fioroni colpisce in particolare perché in contrasto con l'atteggiamento di estrema disponibilità che Milani mostra solo pochi anni prima, quando Fioroni conduce a proprie spese scavi in diversi fondi tarquiniesi, in particolare quelli nelle necropoli villanoviane e orientalizzanti sui Poggi a nord e nord-est dei Monterozzi tra il 1904 e il 1906, al punto da intervenire in aiuto del possidente al quale fa ottenere una proroga eccezionale per la prosecuzione delle ricerche. ${ }^{14}$ Un atteggiamento inizialmente dovuto, oltre che alle maniere deferenti di Fioroni, alla sua appartenenza a una famiglia di un certo prestigio e ai benefici che dalla sua intensa attività di scavatore e commerciante di oggetti archeologici potevano derivare alla Soprintendenza e al Museo.

11. ASAT, pos. A/21, prot. 1313, part. 672. La documentazione consente di correggere quanto indicato nella memoria dattiloscritta del figlio di Fioroni, riguardo al periodo di esecuzione degli acquerelli e già in bibliografia (Colonna 1984, p. 75 e Sarti 2017b, 122). Vedi anche Cuniglio in questo dossier.

12. Lubtchansky 2017a, p. 34-35, Sarti 2017a, p. 38-39.

13. Così F. Barnabei, Barnabei - Delpino 1991, p. 219-220.

14. ASAT, pos. F/9, prot. 1904 
Le copie ad acquerello delle pitture funerarie etrusche nel Museo delle Antichità Etrusche e Italiche 408 Claudia Carlucci e Laura Michetti

Tutto questo muta dunque in relazione alla questione delle copie delle pitture funerarie:

Firenze 28 settembre 1911

A S.E. il Ministro della P.I. Roma

Vengo informato dal custode dei monumenti che il Sig. ${ }^{r}$ V. Fioroni ha continuato fino a pochi giorni fa il lavoro di riproduzione ad acquerello dei dipinti delle tombe etrusche. Già con lettera del 3 luglio prot. 1313 ho richiamato l'attenzione dell'E.V. sopra tale concessione fatta al Sig. ${ }^{\mathrm{r}}$ Fioroni dall'Ufficio dei Monumenti di Roma, ad insaputa di questa Soprintendenza. A tal proposito facevo notare nella lettera citata oltre il servizio gravoso e non ricompensato del custode locale per la sorveglianza, il danno che ne derivava alle pitture stesse per guasti eventuali, durante tale lavoro. Ho inoltre richiamato all'attenzione dell'E.V. come tale questione sia collegata col progetto della costituenda Galleria in fac-simile della pittura etrusca già approvato da codesto ministero. Attuandosi tale progetto mi sembrano dannose queste concessioni a privati, che hanno puramente uno scopo commerciale o di speculazione industriale, poiché con grande vantaggio delle pitture originali soggette a continui danni, i privati ed i musei esteri potranno ritrarre le riproduzioni dalla copia campione che se ne eseguirà per la nostra Galleria.

Attendo una risposta intorno a tale questione rimasta finora insoluta.

Il Soprintendente

Luigi A. Milani. ${ }^{15}$

L'irritazione di Milani, già emersa in relazione alla difficile trattativa per l'acquisto dei materiali villanoviani, si accresce quando il Fioroni gli propone di acquistare le tavole fino a quel momento realizzate dal suo pittore a condizione di poterne trarre copie per sé. Milani risponderà solo un anno dopo, il 15 dicembre 1912, in maniera frettolosa e sprezzante, dopo aver consultato il disegnatore Augusto Guido Gatti che a sua volta aveva iniziato il lavoro di riproduzione delle tombe dipinte tarquiniesi. ${ }^{16}$ La risposta del Gatti al quesito se possano essergli utili le tavole del "pittore di Fioroni» per realizzare le proprie copie, merita di essere segnalata per il giudizio severo che egli esprime sull'operato, sul metodo e i risultati raggiunti da quel pittore:

Rispondendo alla Sua del 27 corrente le faccio notare che in quanto al lavoro che viene eseguito nelle tombe per conto del Sig. Fioroni, nulla nuoce al compimento della mia missione.

In quanto poi alla proposta di fare acquisto degli acquerelli non lo credo opportuno: ormai Ella conosce bene il mio sistema adottato fino ad oggi per le riproduzioni delle pitture murali, così mi troverei non poco imbarazzato il dovere copiare da tavole che come forma delle figure trovo mancante, e punto d'accordo sono per la sua intonazione.

Intanto mi riserbo al prossimo mio ritorno poterle fornire i prezzi delle tavole di ogni singola tomba. Il Disegnatore Guido Gatti. ${ }^{17}$

Laura M. Michetti

\section{«IL PITTORE DI FIORONI»: TECNICA E STILE}

Riguardo all'esecutore degli acquerelli, in assenza di dati nei documenti fiorentini, in cui - come già sottolineato - è citato solo con l'indicazione generica di «il pittore» o «l'artista», le ricerche sulla sua figura hanno dovuto necessariamente basarsi sull'unico documento d'archivio in cui è menzionato in maniera diretta, il citato dattiloscritto con la breve biografia di Vincenzo Fioroni stilata dal figlio. Purtroppo i due unici indizi in essa contenuti, il nome Elio D'Alessandris e il riferimento all'Accademia di Belle Arti di Roma, non sono stati sufficienti per reperirne informazioni né presso l'Archivio dell'Accademia né presso altre istituzioni artistiche romane.

Una possibile prova a favore del fatto che il pittore chiamato Elio D'Alessandris dal figlio di Fioroni fosse attivo nell'ambiente romano, potrebbe indirettamente essere confermato dalla presenza segnalata da Matteo Piccioni negli elenchi della Guida Monaci relativi all'anno 1915 di un Lelio $\mathrm{D}^{\prime}$ Alessandri ${ }^{18}$ che svolgeva la propria atti-
15. ASAT, pos. A/21, prot. 1966, concessione di riprodurre i dipinti delle tombe etrusche a Corneto-Tarquinia.

16. Sarti 2017a; Cuniglio 2017 e Cuniglio - Sarti in questo dossier.
17. ASAT, pos. X/C, prot. 392, riproduzioni delle tombe dipinte. 18. Non Elia d'Alessandri come indicato in Sarti 2017b, p. 122 
vità di pittore in uno studio sito a Roma in Borgo Angelico 95. Infatti non appare inverosimile che il nome indicato dal figlio di Fioroni possa essere stato parzialmente deformato nel testo trasmesso a Massimo Pallottino tramite la moglie, affidato com'era esclusivamente alle memorie di ragazzo affascinato dall'attività del padre.

Nessun rapporto sembra invece riscontrabile con l'ambiente tarquiniese in cui operava Fioroni: ricerche svolte presso i registri del Comune di Tarquinia e della Diocesi di Civitavecchia-Tarquinia non registrano infatti nessun nominativo analogo a quello indicato dal figlio di Fioroni. ${ }^{19}$

Ignoriamo purtroppo quali rapporti ci fossero tra Fioroni e il pittore e per quale motivo la scelta fosse caduta proprio su questo personaggio. ${ }^{20}$ Data l'impossibilità di avere al momento certezze sulla possibile corrispondenza tra il pittore al servizio di Fioroni e i personaggi menzionati nella nota biografica, in seguito si farà riferimento al copista esclusivamente con l'indicazione «il pittore di Fioroni» e alle riproduzioni con il termine "copie Fioroni».

Sulle 52 tavole riproducenti le 24 tombe di Tarquinia il pittore ha riportato in alto a sinistra l'indicazione della località, Corneto Tarquinia, in alto al centro e a destra rispettivamente la denominazione della tomba e il relativo numero romano progressivo, da I a XXIV (cfr. fig. 1-5, 7, 9-11), a essa assegnato negli anni dell'esecuzione delle copie tra il 1911 ed il 1912 (tab. 1).

\begin{tabular}{|c|c|c|c|c|c|c|c|c|c|}
\hline & $\begin{array}{l}\text { Pittore di } \\
\text { Fioroni }\end{array}$ & Morani & Gatti & Ajelli & & $\begin{array}{l}\text { Pittore di } \\
\text { Fioroni }\end{array}$ & Morani & Gatti & Ajelli \\
\hline I & $\begin{array}{l}\text { T. Caccia } \\
\text { e Pesca }\end{array}$ & $\begin{array}{l}\text { T. Caccia } \\
\text { e Pesca }\end{array}$ & $\begin{array}{l}\text { T. Caccia } \\
\text { e Pesca }\end{array}$ & $\begin{array}{l}\text { T. Caccia } \\
\text { e Pesca }\end{array}$ & XIII & $\begin{array}{l}\text { T. Polifemo } \\
\text { (Orco I e II) }\end{array}$ & $\begin{array}{l}\text { XIV T. Orco } \\
\text { (I e II) }\end{array}$ & T. Orco & T. Orco \\
\hline II & T. Leonesse & T. Leonesse & & T. Leonesse & XIV & $\begin{array}{l}\text { T. Vasi } \\
\text { Dipinti }\end{array}$ & $\begin{array}{l}\text { XV T. Vasi } \\
\text { Dipinti }\end{array}$ & $\begin{array}{l}\text { T. Vasi } \\
\text { Dipinti }\end{array}$ & $\begin{array}{l}\text { T. Vasi } \\
\text { Dipinti }\end{array}$ \\
\hline III & T. Pulcella & T. Pulcella & & & $X V$ & T. Vecchio & $\begin{array}{l}\text { XVI } \\
\text { T. Vecchio }\end{array}$ & & \\
\hline IV & $\begin{array}{l}\text { T. Caccia al } \\
\text { Cinghiale } \\
\text { (Querciola I) }\end{array}$ & $\begin{array}{l}\text { T. Querciola } \\
\text { (Caccia al } \\
\text { Cinghiale) } \\
\end{array}$ & & & XVI & T. Morente & $\begin{array}{l}\text { T. Moribondo } \\
\text { (Morente) }\end{array}$ & & \\
\hline V & T. Leopardi & T. Leopardi & & & XVII & T. Iscrizioni & $\begin{array}{l}\text { XVIII } \\
\text { T. Iscrizioni }\end{array}$ & & \\
\hline VI & $\begin{array}{l}\text { T. Letto } \\
\text { Funebre }\end{array}$ & $\begin{array}{l}\text { T. Letto } \\
\text { Funebre }\end{array}$ & & & XVIII & T. Bighe & XXII T. Bighe & & \\
\hline VII & $\begin{array}{l}\text { T. Convito } \\
\text { (Triclinio) }\end{array}$ & T. Triclinio & & T. Triclinio & XIX & T. Barone & T. Barone & & \\
\hline VIII & T. Baccanti & T. Baccanti & & & $\mathrm{XX}$ & T. Tori & XXV T. Tori & T. Tori & T. Tori \\
\hline IX & T. Morto & X T. Morto & & & XXI & $\begin{array}{l}\text { T. Secondi } \\
\text { Archi } \\
\text { (Pulcinella) }\end{array}$ & $\begin{array}{l}\text { XXIV } \\
\text { T. Pulcinella }\end{array}$ & & \\
\hline $\mathrm{X}$ & T. Tifone & XI T. Tifone & & T. Tifone & XXII & $\begin{array}{l}\text { T. Due } \\
\text { Bighe } \\
\text { (Francesca } \\
\text { Giustiniani) }\end{array}$ & $\begin{array}{l}\text { XX } \\
\text { T. Giustiniani } \\
\text { (Francesca) }\end{array}$ & & $\begin{array}{l}\text { T. Francesca } \\
\text { Giustiniani }\end{array}$ \\
\hline XI & T. Scudi & XII T. Scudi & & & XXIII & T. Auguri & T. Auguri & & \\
\hline XII & T. Cardinale & $\begin{array}{l}\text { XIII } \\
\text { T. Cardinale }\end{array}$ & $?$ & $\begin{array}{l}\text { T. } \\
\text { Cardinale }\end{array}$ & XXIV & T. Mare & & & \\
\hline
\end{tabular}

19. Ricerche svolte per conto di Luciana Drago da Beatrice Casocavallo, funzionario presso la sede di Tarquinia della Soprintendenza Archeologia, Belle Arti e Paesaggio per l'area metropolitana di Roma, la provincia di Viterbo e l'Etruria meridionale.
20. Un canale tra Vincenzo Fioroni e il pittore potrebbe essere stato l'altro figlio di Fioroni, Mario, noto scultore del Novecento e padre della più celebre artista Giosetta. Egli che, proprio in quegli anni, aveva frequentato il Regio Istituto di Belle Arti legato all'Accademia di San Luca (Pancotto 1997) dove fu allievo di Arturo Martini ed Ettore Ferrari, potrebbe forse aver proposto per l'esecuzione delle copie un compagno di studi, avviato poi a minor fortuna rispetto allo scultore. 
Le copie ad acquerello delle pitture funerarie etrusche nel Museo delle Antichità Etrusche e Italiche

410 Claudia Carlucci e Laura Michetti

Tale sequenza corrisponde largamente all'ordine topografico della distanza dei sepolcri a partire dall'abitato moderno, da tempo consolidato, almeno dal 1878, come mostra la serie di elenchi delle tombe compilati dai diversi «ispettori e custodi delle tombe» editi da Adele Cecchini nel volume dedicato alla storia dei restauri delle tombe tarquiniesi. $^{21}$

Nella numerazione delle tombe sono da segnalare piccole differenze rispetto al numero comunemente a esse assegnato negli anni immediatamente precedenti e successivi all'esecuzione delle copie, come risulta dalla tabella 1 relativa alla comparazione tra i cicli pittorici di Morani dell'Helbig Museum di Copenaghen (1897-1910), quello romano del Museo universitario commissionato da Fioroni (1911-1912), quello fiorentino di Guido Gatti (1912) e quello tarquiniese di Adolfo Ajelli $(1934)^{22}$, e dal confronto con la serie di elenchi delle tombe resi noti da Cecchini (tab. 2$)^{23}$.

\begin{tabular}{|l|l|l|l|l|l|l|l|}
\hline & $\begin{array}{l}\text { Dasti 1910 } \\
\text { (numerazione } \\
\text { corretta) }\end{array}$ & Acquerelli & $\begin{array}{l}\text { Roma } \\
\text { Museo } \\
\text { Sapienza }\end{array}$ & $\begin{array}{l}\text { La Valle } \\
1913\end{array}$ & $\begin{array}{l}\text { Ny } \\
\text { Carlsberg }\end{array}$ & $\begin{array}{l}\text { Ajelli } \\
\text { (s.n.) }\end{array}$ & Gatti \\
\hline $\begin{array}{l}\text { T. Cacciatore } \\
\text { (o Caccia e Pesca) }\end{array}$ & 1 & 1 & I & 1 & I & $\S$ & $\S$ \\
\hline T. Leonesse & 2 & 2 & II & 2 & II & $\S$ & \\
\hline T. Pulcella & 3 & 3 & III & 3 & III & $\S$ & \\
\hline $\begin{array}{l}\text { T. Caccia al cinghiale } \\
\text { (o Querciola I) }\end{array}$ & 4 & 4 & IV & 4 & s.n. & $\S$ & \\
\hline T. Leopardi & 5 & 5 & V & 5 & s.n. & $\S$ & \\
\hline T. Letto funebre & 6 & 6 & VI & 6 & VI & $\S$ & \\
\hline $\begin{array}{l}\text { T. } \text { Triclinio } \\
\text { (o Convito) }\end{array}$ & 7 & 7 & VII & 7 & s.n. & $\S$ & \\
\hline T. Baccanti & 8 & 8 & VIII & 8 & VIII & $\S$ & \\
\hline T. Morto & $9(10)$ & 9 & IX & 9 & X & $\S$ & \\
\hline T. Tifone & $10(11)$ & 10 & X & 10 & XI & $\S$ & \\
\hline $\begin{array}{l}\text { T. Scudi } \\
\text { (o Quattro stanze) }\end{array}$ & $11(12)$ & 11 & XI & 11 & XII & $\S$ & \\
\hline T. Cardinale & $12(13)$ & 12 & XII & 12 & XIII & $\S$ & \\
\hline T. Polifemo (Orco) & $13(14)$ & 13 & XIII & 13 & XIV & $\S$ & $\S$ \\
\hline
\end{tabular}

21. Cecchini 2012, p. 83-84, la presentazione delle tombe secondo la posizione topografica viene seguita anche dal custode Edmondo La Valle nella pianta pubblicata nella sua guida, La Valle 1913. Sulla figura del La Valle, “custode delle tombe", Cecchini 2012, p. 57-58.
22. I cicli pittorici del Gatti, limitati a sole quattro tombe, e di Ajelli non riportano né fanno riferimento ad alcuna numerazione delle tombe.

23. Tra gli elenchi pubblicati in Cecchini 2012, p. 83-84, sono stati scelti i più completi redatti da Luigi Dasti, nelle due versioni del 1878 e 1910, e da Edmondo La Valle, rispettivamente corrispondenti alle relative pubblicazioni (Dasti 1878 e 1910; La Valle 1913). Significativo, per la storia delle scoperte delle tombe tarquiniesi e della loro tutela, appare il numero dei diversi elenchi redatti a partire dal 1878 che testimonia la difficoltà di seguire con ordine e, soprattutto, documentare in tempo reale la scoperta, le chiusure e le riaperture delle tombe in occasione dei ripetuti restauri delle pitture e delle tombe stesse. Emblematico risulta il caso della non meglio identificata Tomba delle Pantere, indicata con il numero IX, nel primo elenco del Dasti del 1878, Cecchini 2012, p. 83, che non può essere identificata con la ben nota tomba delle Pantere, scoperta solo nel 1970, ma che è possibile collocare topograficamente tra le tombe dei Baccanti e del Morto. Di questa tomba non si farà più menzione nel secondo elenco Dasti 1910, ma in La Valle 1913, vi è l'annotazione che la tomba n. 9 "più non esiste essendo stata richiusa», Cecchini 2012, p. 58. 


\begin{tabular}{|c|c|c|c|c|c|c|c|}
\hline T. Vasi Dipinti & $14(15)$ & 14 & XIV & 14 & $\mathrm{XV}$ & $\S$ & $\S$ \\
\hline T. Vecchio & $15(16)$ & 15 & XV & 15 & XVI & $\S$ & \\
\hline T. Morente & $16(17)$ & 16 & XVI & 16 & s.n. & $\S$ & \\
\hline T. Iscrizioni & $17(18)$ & 17 & XVII & 17 & XVIII & & \\
\hline $\begin{array}{l}\text { T. Bighe (o } \\
\text { Stackelberg) }\end{array}$ & $18(20)$ & 18 & XVIII & 21 & XXII & $\S$ & \\
\hline $\begin{array}{l}\text { T. Barone } \\
\text { (o Corsa dei cavalli) }\end{array}$ & $19(19)$ & 19 & XIX & 18 & XIX & $\S$ & \\
\hline T. Tori & non in elenco & 20 & $\mathrm{XX}$ & s.n. & XXV & $\S$ & $\S$ \\
\hline $\begin{array}{l}\text { T. Mare } \\
\text { (o Due stanze) }\end{array}$ & $20(21)$ & & XXIV & 20 & & & \\
\hline $\begin{array}{l}\text { T. Francesca } \\
\text { Giustiniani } \\
\text { (o Due bighe) }\end{array}$ & $21(22)$ & 22 & XXII & 19 & $\mathrm{xx}$ & $\S$ & \\
\hline T. Auguri & $22(28)$ & 23 & XXIII & 22 & XXIII & $\S$ & \\
\hline T. ai Secondi archi & $23(24)$ & & & & & & \\
\hline $\begin{array}{l}\text { T. Pulcinella } \\
\text { (o Bajetti) }\end{array}$ & 24 richiusa $(23)$ & 24 & XXI & 23 & XXIV & $\S$ & \\
\hline T. Porta di bronzo & 25 richiusa $(25)$ & & & & & $\S$ & \\
\hline senza nome & $(26)$ & & & & & & \\
\hline senza nome & $(27)$ & & & & & & \\
\hline T. Festoni & & & & & & $\S$ & \\
\hline T. Tarantola & & & & & & $\S$ & \\
\hline T. Bruschi & & & & & & $\S$ & \\
\hline T. Olimpiadi & & & & & & $\S$ & \\
\hline T. Nave & & & & & & $\S$ & \\
\hline
\end{tabular}

Elaborazione da Cecchini 2012, p. 83-84.

Le differenze nella numerazione di maggiore interesse riguardano il confronto tra la serie romana e quella di Copenaghen, le più vicine tra loro anche per completezza. Esse infatti riproducono le stesse 23 tombe tarquiniesi, a cui il ciclo commissionato da Fioroni, come già sottolineato l'unico tra quelli realizzati tra fine Ottocento e primi decenni del Novecento, aggiunge anche la Tomba del Mare, indicata con il numero XXIV, non conforme a nessuna delle serie di numerazione delle tombe in uso a partire dal 1878. Non sappiamo se la tomba non sia stata riprodotta dall'équipe di Morani perché non accessibile negli anni in cui era al lavoro nella necropoli (ovvero tra il 1897 e il 1903, anni per i quali non è disponibile alcun elenco specifico) o per altre motivazioni o scelte dei pittori o di Helbig e/o Jacobsen.

Solo 11 delle 23 tombe copiate in entrambi i cicli riportano la stessa numerazione (tab. 1), in alcuni casi neanche conforme agli elenchi redatti in tre momenti diversi del 1878 e del 1910 dal Dasti e del 1913 da parte di Edmondo La Valle (tab. 2). Il motivo di tale difformità è senza dubbio da attribuire, come già osservato da Cecchini ${ }^{24}$, all'uso di numerare le tombe sulla base dello scavo, chiusura e riapertura delle stesse, risultando così piuttosto variabile. Inoltre è da rilevare che non sempre le modifiche nella sequenza erano puntualmente registrate in documenti d'archivio o in pubblicazioni a stampa.

Da quanto risulta da una lettera del Gatti a Milani del 25 maggio 191225, sembrerebbe che «il pittore di Fioroni» possa aver eseguito le copie ad acquerello in scala ridotta direttamente nelle tombe, mentre dalla nota biografica del figlio di

24. Cecchini 2012, p. 32-34, p. 49, nota 143, p. 86, nota 29.

25. ASAT, pos. 10/C, prot. 392, Corneto Tarquinia. Riproduzioni delle pitture etrusche. 
Le copie ad acquerello delle pitture funerarie etrusche nel Museo delle Antichità Etrusche e Italiche

412 Claudia Carlucci e Laura Michetti

Fioroni ricaviamo la scarna notizia circa la modalità di lavoro utilizzata: «portando la luce del sole nelle tombe, con un sistema di specchi, e con lampade a carburo». Appare però verosimile, anche tenendo conto della varietà inconsueta delle scale di rappresentazione utilizzate, per le quali non è agevole trovare spiegazioni plausibili ${ }^{26}$, che egli abbia prodotto preliminarmente degli studi preparatori a matita sulla base di fotografie, successivamente integrati direttamente nelle tombe con pennello e colori. È ipotizzabile anche che le bozze preparatorie non siano state consegnate a Fioroni, come potrebbe essere nel caso della Tomba del Polifemo, cioè la Tomba dell'Orco, a cui il pittore stava lavorando con un orario quotidiano di tre ore secondo quanto riferito nella suddetta lettera del Gatti.

La redazione finale delle copie appare dunque il risultato del montaggio di due diversi momenti: il primo consistente nella copia a matita della foto, come dimostrano la presenza di una quadrettatura, visibile, per esempio, nella Tomba del Morto (fig. 2, 5 e 6) e nella Tomba degli Scudi (fig. 7-8), i contorni ben delineati della matita, in particolare nella definizione delle lacune ed altri dettagli; il secondo con la stesura del colore in base agli appunti presi direttamente all'interno delle tombe, secondo una prassi ormai consolidata nella realizzazione delle copie. Nel complesso il lavoro del pittore di Fioroni risulta diseguale. A fronte di acquerelli di un certo pregio come le riproduzioni della Tomba dei Leopardi (fig. 9), nella copia della Tomba della Caccia al Cinghiale (Querciola I) (fig. 10), il pittore realizza una versione tra le più trascurate e superficiali della sua serie, che nell'insieme si mantiene conforme alle fotografie del tempo, ma nella quale elimina tutti i dettagli, come si nota soprattutto nel ritratto molto stilizzato del banchettante a destra della parete di fondo (fig. 11).

Rispetto alle altre serie, quelle del Museo della Sapienza sono più scolastiche, denunciando una mano non sempre sicura anche nella trascrizione delle iscrizioni, oltre che un'attitudine a comporre in maniera standardizzata i particolari, tentando di dare una visione d'insieme dello stato della tomba, proponendo il punto di vista dell'ipotetico

26. In alcuni casi non è stata utilizzata la stessa scala neanche per la riproduzione delle pareti della stessa tomba, come nelle tombe del Cardinale e del Tifone. Sono state impiegate fino a 20 scale differenti che si attestano tra 1:0,08 e 1:0,137.

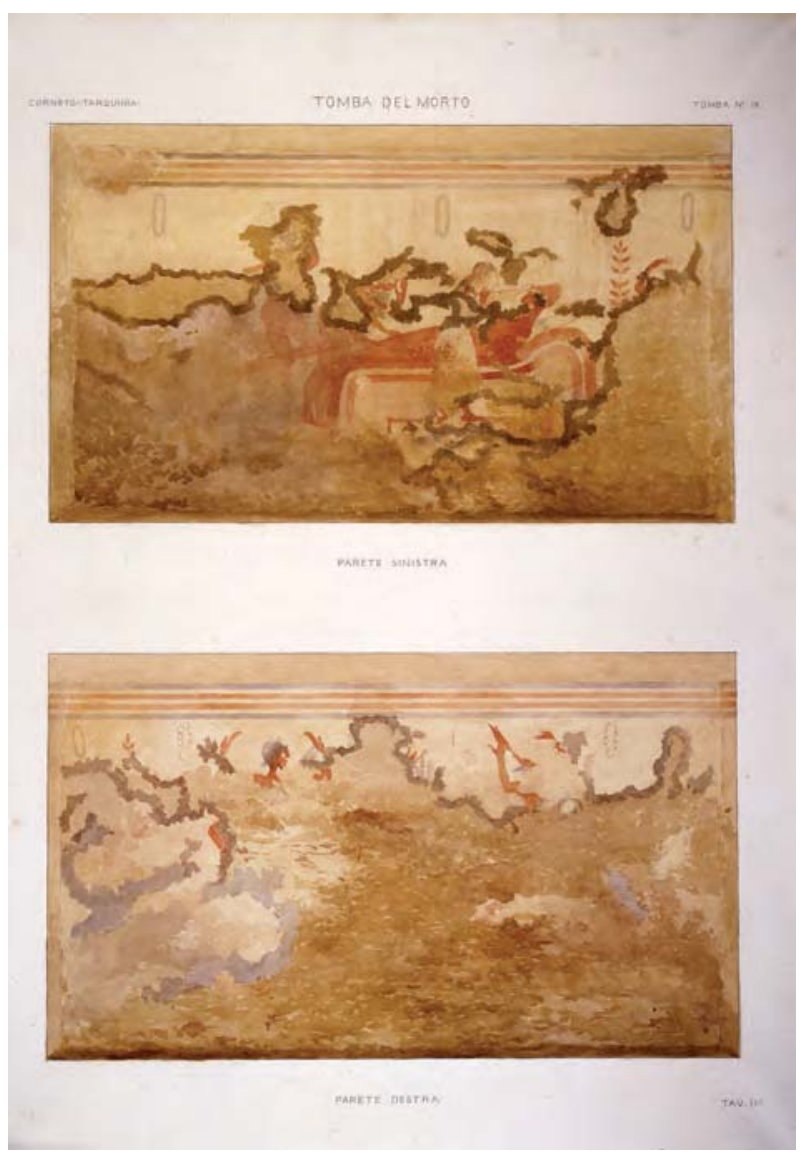

Fig. 5 - Acquerello, Tomba del Morto, tomba n. IX, tav. II. (C) AMAEIS.

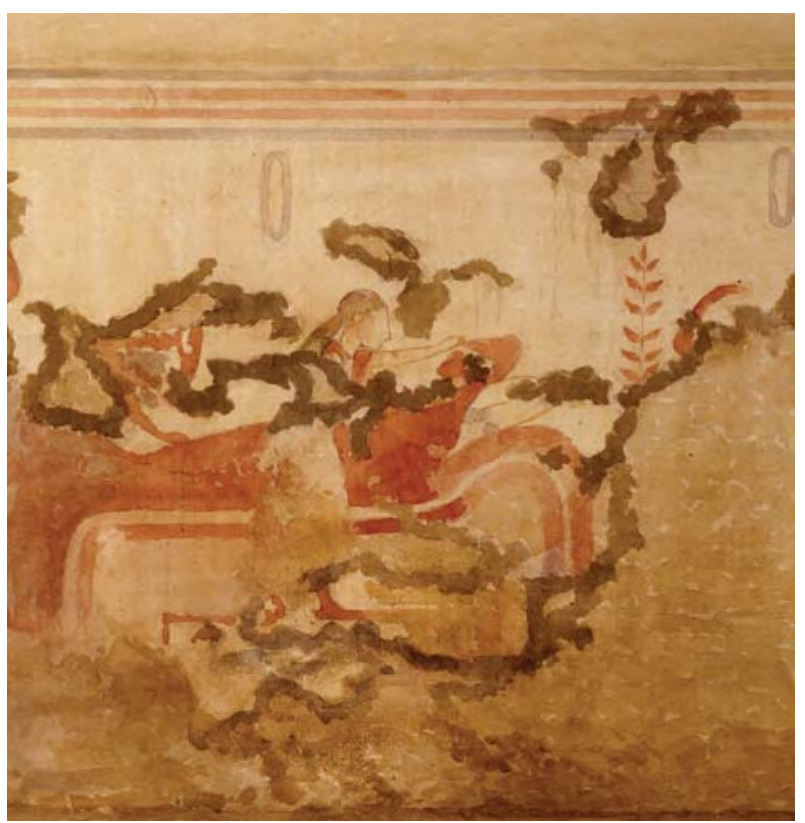

Fig. 6 - Acquerello, Tomba del Morto, particolare con tracce della quadrettatura a matita. (c) AMAEIS. 
osservatore, piuttosto che un'analisi minuziosa dei dettagli. Questo stile "impressionistico", tendente a ricreare una visione verosimile dell'interno della tomba, denuncia un pittore ancora legato ai modi del paesaggismo ottocentesco, come si evince nella

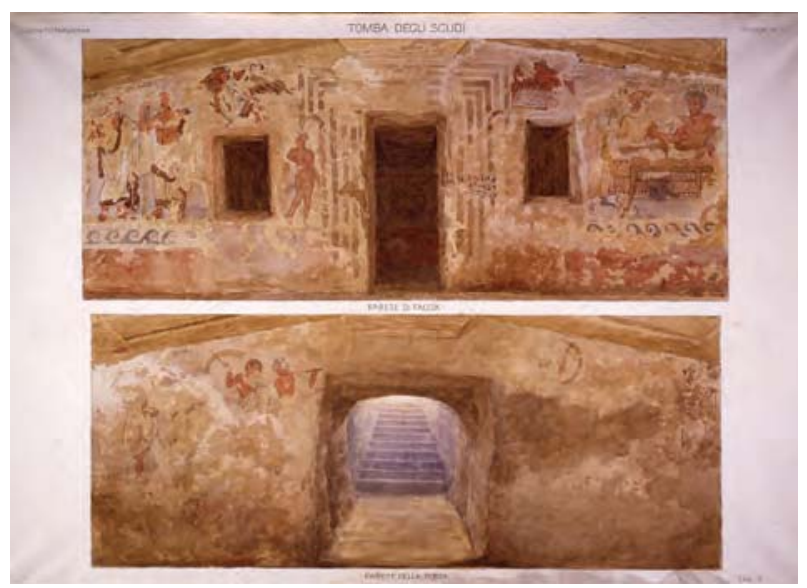

Fig. 7 - Acquerello, Tomba degli Scudi, tomba n. XI, tav. Ia. (C) AMAEIS.

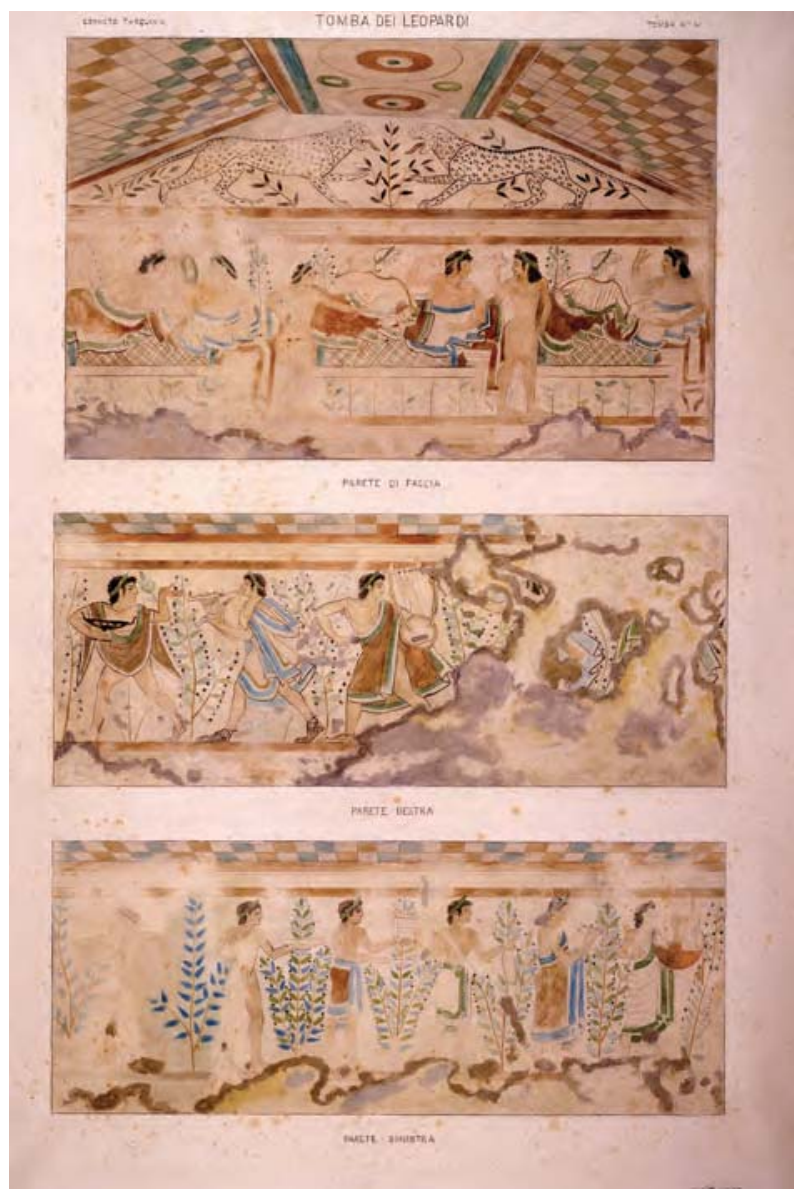

Fig. 9 - Acquerello, Tomba dei Leopardi, tomba n. V. (C) AMAEIS. riproduzione degli esterni in alcune tavole, certamente non in linea con le tendenze dell'arte coeva più avanzata.

L'aspetto più rigoroso di documentazione analitica delle pitture è maggiormente riscontrabile

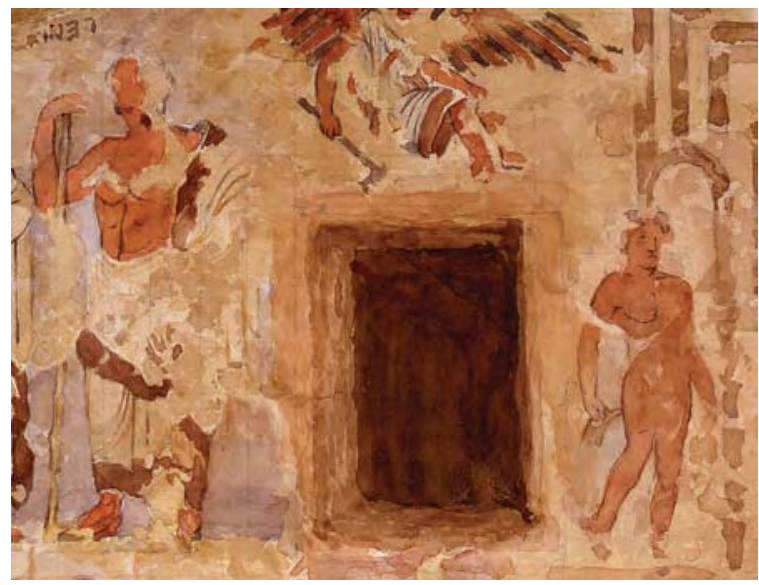

Fig. 8 - Acquerello, Tomba degli Scudi, particolare con tracce del disegno preparatorio a matita. (c) AMAEIS.

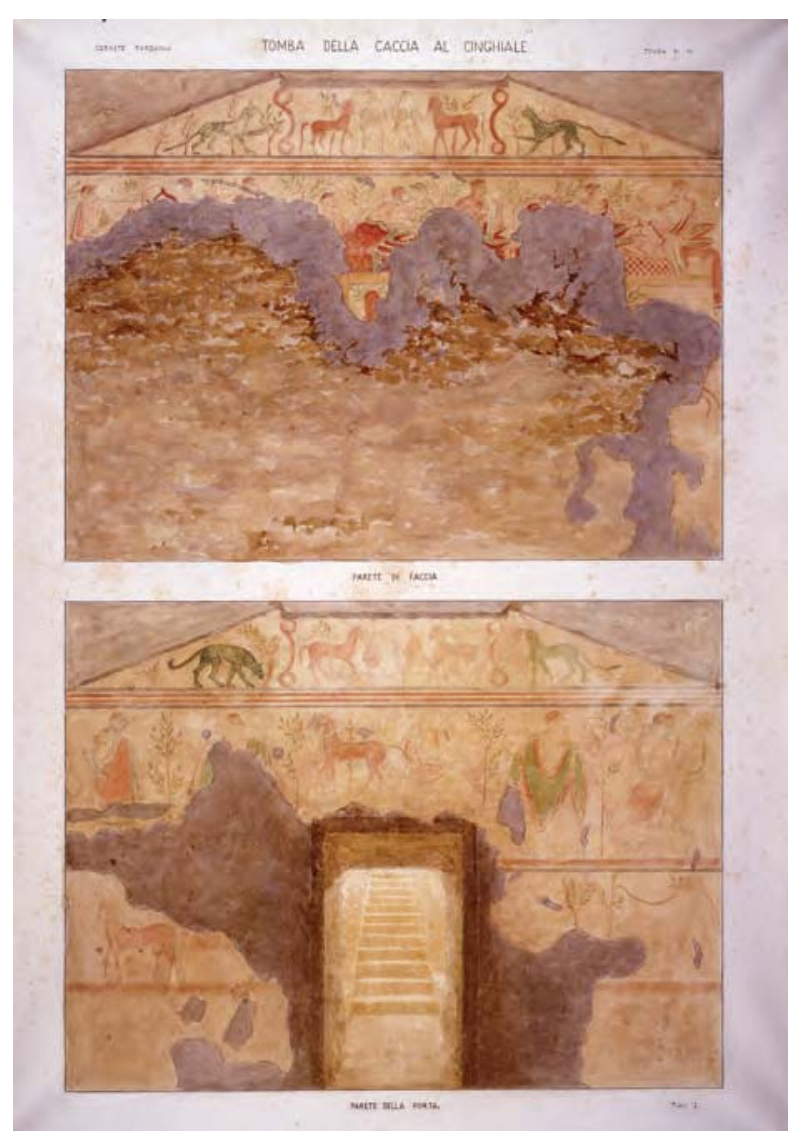

Fig. 10 - Acquerello, Tomba della Caccia al Cinghiale (Querciola I), tomba n. IV, tav. I. (c) AMAEIS. 


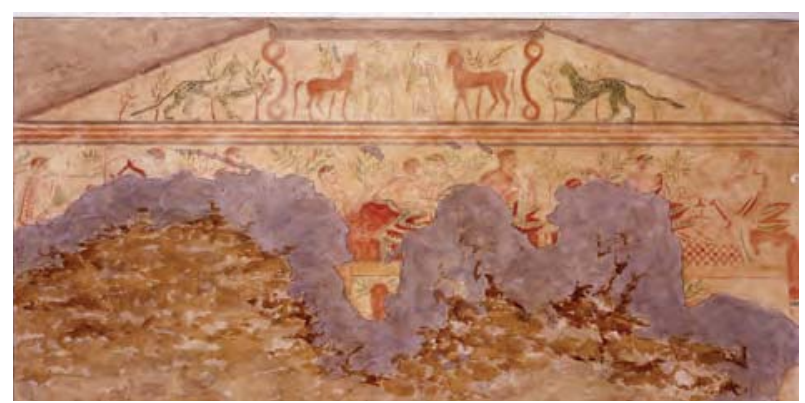

Fig. 11 - Acquerello, Tomba della Caccia al Cinghiale (Querciola I), tomba n. IV, tav. I.

(c) AMAEIS.

nelle copie Morani-Helbig, vista anche la destinazione più prettamente scientifica del prodotto finale, nonché il passaggio alle dimensioni al vero dei facsimile.

Per valutare l'attendibilità delle riproduzioni del "pittore di Fioroni» a confronto con le altre copie realizzate tra Ottocento e Novecento, può essere utile proporre, a titolo di esempio, alcune osservazioni su alcune tra le tombe riprodotte che presentano maggiore interesse, tenendo conto anche della relativa documentazione fotografica dell'epoca.

La Tomba della Caccia e Pesca (fig. 12) rappresenta uno dei migliori esempi per una comparazione. Scoperta nel 1873, fu riprodotta per la prima volta da cromolitografie di Gregorio Mariani nel 1885 in Monumenti inediti per iniziativa dell'Istituto di Corrispondenza Archeologica. ${ }^{27}$ I disegni di Mariani, conservati ancora presso l'Istituto Archeologico Germanico, e utilizzati per la preparazione nel 1896 dei facsimile nella Ny Carlsberg Glyptotek di Copenaghen, presentano colori e dettagli apparentemente realistici in relazione al degrado della tomba al momento della scoperta, mentre solo alcuni dei dettagli relativi allo stato di conservazione compaiono nei facsimile di Copenaghen, che mostrano la tendenza a compiere integrazioni nelle porzioni dei dipinti in cui la lettura dell'immagine non lascia adito a dubbi.

Rispetto ai disegni e alle riproduzioni fotografiche di Mariani, le copie Fioroni riproducono per intero la grande lacuna sull'architrave della

27. Lubtchansky 2017b e Capoferro in questo dossier.

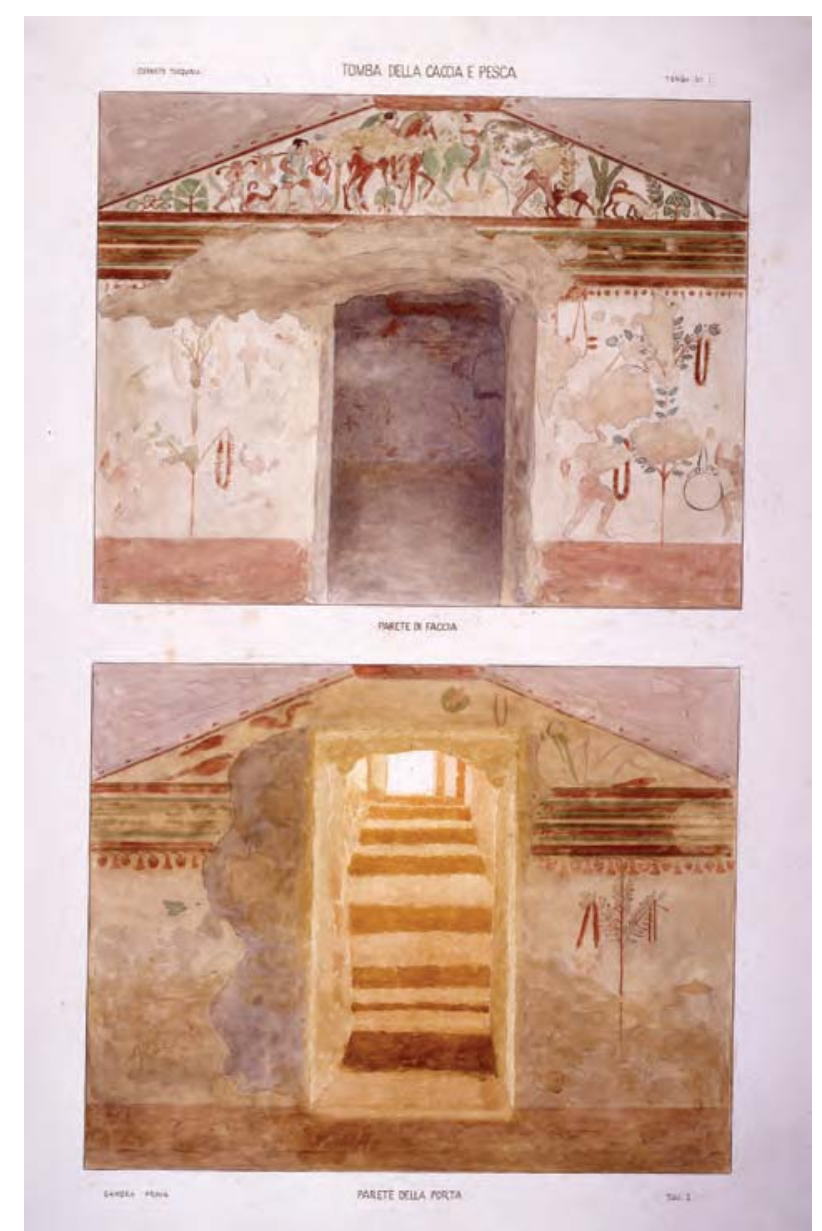

Fig. 12 - Acquerello, Tomba della Caccia e Pesca, tomba n. I, tav. I. (C) AMAEIS.

porta che dà sulla camera interna e sembrano, nell'insieme e relativamente ai dettagli, affidabili quanto il lavoro di Mariani a confronto delle pitture originali. A differenza di Mariani, il pittore di Fioroni offre una prospettiva quasi tridimensionale degli ambienti della tomba nel suo complesso, compresa la veduta dei soffitti, verosimilmente tenendo conto delle riprese fotografiche allora in circolazione, probabilmente soprattutto quelle di Romualdo Moscioni ${ }^{28}$. Mariani invece scompone il disegno della seconda camera, l'unico pervenuto, in pareti e loro porzioni, aggiungendo lateralmente la mezza paratia ai lati della porta. Alessandro Morani, che realizzerà per la Ny Carlsberg le riproduzioni delle parti mancanti nelle copie Mariani (le

28. Cecchini 2012, p. 53-56; anche in La Valle 1913 venne impiegata la documentazione fotografica di Moscioni Cecchini 2012, p. 58. 
pareti d'ingresso di entrambe le camere e le pareti laterali della prima camera), inserite nella «bella copia" realizzata nel 1908, scompone l'intero complesso delle pitture in soffitti, pareti e pianta. ${ }^{29}$

La copia che merita forse più di una menzione è quella della Tomba del Mare (fig. 4). Per questa riproduzione è importante sottolineare non tanto la sua qualità, quanto la sua importanza come documento, testimonianza unica delle condizioni della tomba all'inizio del Novecento. Infatti, le sole riproduzioni note della tomba risalgono alla prima metà del XIX secolo, successivamente alla scoperta nel 1825, ad opera di Otto von Stackelberg e Henri Labrouste. ${ }^{30}$ Come per le altre tombe, l'inquadratura è prospettica e frontale, per dare una visione di insieme delle camere. L'acquerello appare accurato, sebbene non particolareggiato, sia nel tracciare la decorazione dei fregi a fasce cromatiche,

\section{Bibliografia}

\section{Archivi}

ASAT $=$ Archivio Storico della ex Soprintendenza Archeologia della Toscana, Firenze

AMAEIS = Archivio del Museo delle Antichità Etrusche e Italiche della Sapienza Università di Roma

\section{Bibliografia secondaria}

Banti 1967 = L. Banti, Disegni di tombe e monumenti etruschi fra il 1825 e il 1830: l'architetto Henri Labrouste, in StEtr, 35, 1967, p. 575-593.

Barnabei - Delpino 1991 = M. Barnabei, F. Delpino $(\mathrm{a} \mathrm{cu}-$ ra di), Le "Memorie di un archeologo" di Felice Barnabei, Roma, 1991.

Capoferro - Renzetti 2017 = A. Capoferro, S. Renzetti (a cura di), L'Etruria di Alessandro Morani: riproduzioni di pitture etrusche dalle collezioni dell'Istituto Svedese di Studi Classici a Roma, Firenze, 2017.

Cecchini 2012 = A. Cecchini, Le tombe dipinte di Tarquinia. Vicenda conservativa, restauri, tecnica di esecuzione, Firenze, 2012.

29. Capoferro - Renzetti 2017, p. 233-235, per gli acquerelli preparatori alle «belle copie»; Moltesen 2017, p. 143, per il riferimento alle «belle copie»; Capoferro in questo dossier.

30. O.M. von Stackelberg, A. Kestner, Unedierte Gräber von Corneto, s.l., s.d., tav. 34-36; Banti 1967, p. 582-586. sia nel registrare lacune e superfici deteriorate.

Infine, un discorso a parte sarebbe necessario per le riproduzioni della Tomba della Scimmia di Chiusi e della Tomba Golini I di Orvieto che differiscono sensibilmente per stile dalle copie tarquiniesi, e sulle quali ci proponiamo di realizzare un approfondimento di carattere comparativo in futuro. In assenza di documentazione riguardo a queste due serie di copie, è possibile solo avanzare delle ipotesi sulle circostanze della loro acquisizione. Infatti Fioroni potrebbe esserne venuto in possesso successivamente, con il desiderio di completare la serie delle pitture funerarie, oppure potrebbe aver ingaggiato negli stessi anni diversi pittori per lavorare ad Orvieto e Chiusi in parallelo con l'autore che stava lavorando a Tarquinia.

Claudia Carlucci

Colonna 1984 = G. Colonna, Il Museo di Etruscologia, in La cultura scientifica a Roma 1870-1911, Vicenza, 1984, p. 69-77.

Colonna 1993 = G. Colonna, Il Museo delle Antichità Etrusche e Italiche, in M. Barbanera, L. Venafro (a cura di), I Musei dell'Università "La Sapienza", Roma, 1993, p. 279-291.

Cristofani 1971 = M. Cristofani, Monumenti della pittura antica scoperti in Italia, sezione prima. La pittura etrusca. Tarquinii, fasc. V, Le pitture della Tomba del Tifone, Roma, 1971.

Cuniglio 2017 = L. Cuniglio, L'opera di Augusto Guido Gatti per le riproduzioni della pittura etrusca: materiali e tecniche, in Cuniglio - Lubtchansky - Sarti 2017, p. 51-58.

Cuniglio - Lubtchansky - Sarti 2017 = L. Cuniglio, N. Lubtchansky, S. Sarti, Dipingere l'Etruria. Le riproduzioni delle pitture etrusche di Augusto Guido Gatti, Venosa, 2017.

Dasti 1878 = L. Dasti, Notizie Storiche Archeologiche di Corneto e Tarquinia, Roma, 1878.

Dasti 1910 = L. Dasti, Notizie Storiche Archeologiche di Tarquinia e Corneto, seconda edizione ristampata per cura di Gustavo Scotti, Tarquinia, 1910. 
Le copie ad acquerello delle pitture funerarie etrusche nel Museo delle Antichità Etrusche e Italiche 416 Claudia Carlucci e Laura Michetti

Drago Troccoli 2005 = L. Drago Troccoli (a cura di), Il Museo delle Antichità Etrusche e Italiche, I. La protostoria, Roma, 2005.

La Valle 1913 = E. La Valle, Corneto Monumentale e la Necropoli Etrusca Tarquiniese, Corneto-Tarquinia, 1913.

Lubtchansky 2017a $=$ N. Lubtchansky, Documentation graphique et musées de peinture étrusque, in Cuniglio Lubtchansky - Sarti 2017, p. 17-35.

Lubtchansky 2017b = N. Lubtchansky, Tarquinia (Viterbo). Tomba della Caccia e Pesca, in Cuniglio - Lubtchansky Sarti 2017, p. 147-150.

Moltesen 2017 = Mette Moltesen, Alessandro Morani e le "belle copie" delle pitture funerarie etrusche di Chiusi e Tarquinia, in Capoferro - Renzetti 2017, p. 129-145.
Morandi 1983 = A. Morandi, Monumenti della pittura antica scoperti in Italia, sezione prima. La pittura etrusca. Tarquinii, fasc. VI, Le pitture della Tomba del Cardinale, Roma, 1983.

Pancotto 1997 = P.P. Pancotto, Fioroni Mario, in Dizionario Biografico degli Italiani, vol. 48, Roma, 1997.

Sarti $2017 \mathrm{a}=$ S. Sarti, La Galleria della pittura etrusca in facsimile a Firenze, in Cuniglio - Lubtchansky - Sarti 2017, p. 37-58.

Sarti $2017 b=$ S. Sarti, Orvieto (Terni), Tomba Golini I (dei Velii, dei Settecamini), in Cuniglio - Lubtchansky - Sarti 2017, p. 109-122. 
Le ultime acquisizioni dal teatro di Terracina e l'eccezionale iscrizione del triumviro M. Emilio Lepido 518 Nicoletta Cassieri, Gian Luca Gregori e Jean-Baptise Refalo-Bistagne

Lugli 1926 = G. Lugli, Anxur-Tarracina, Roma, 1926 (Forma Italiae, I, 1).

Melchor Gil 2017 = E. Melchor Gil, Los origenes del patronato civico en las provincias hispanas: desde Cneo Pompeyo Magno al triunviro Marco Emilio Lépido, in RSA, 47, 2017, p. 35-58.

Melchor Gil 2018 = E. Melchor Gil, El patronato cívico en la Hispania romana, Sevilla, 2018.

Mesolella 2012 = G. Mesolella, La decorazione architettonica di Minturnae Formiae Tarracina: l'età augustea e giulio-claudia, Roma, 2012.

Paci $2014=$ G. Paci, Liberte e schiave a teatro e all'anfiteatro nel Piceno, in C. Zaccaria (a cura di), L'epigrafia dei porti, Trieste, 2014, p. 275-288.

Pina Polo 2018 = F. Pina Polo, Magistrates without pedigree: the consules suffecti of the Triumviral Age, in JRS, 108, 2018, p. 99-114.

Roddaz $2003=$ J.-M. Roddaz, Octavien-Auguste et les soldats perdus de Lépide, in J.-P. Bost, J.-M. Roddaz, F. Tassaux (a cura di), Mélanges offerts à Louis Maurin. Itinéraire de Saintes à Dougga, Bordeaux, 2003, p. 189-203.

Rohr Vio 2004 = F. Rohr Vio, Marco Emilio Lepido tra memoria e oblio nelle Historiae di Velleio Patercolo, in RCCM, 46-2, 2004, p. 277-301.

Rohr Vio 2007 = F. Rohr Vio, Recensione a Allely 2004, in RCCM, 49, 2007, p. 173-176.

Ronchi 2017 = D. Ronchi, La colonia di Circeii. Dal tardo arcaismo alla colonia di Cesare padre: santuari ed evidenze monumentali, Pisa, 2017.

Schneider $1986=$ R.M. Schneider, Bunte Barbaren. Orientalenstatuen aus farbigen Marmor in der römischen Repräsentationskunst, Worms, 1986.

Schumacher $1985=$ L. Schumacher, Die imperatorischen Akklamationen der Triumvirn und die auspicia des Augustus, in Historia, 34-2, 1985, p. 191-222.
Simpson 2006 = C.J. Simpson, M. Aemilius Lepidus, in Latomus, 65-3, 2006, p. 628-633.

Solin $1981=\mathrm{H}$. Solin, Caesar und P. Clodius Pulcher in Terracina, in ZPE, 43, 1981, p. 357-361.

Syme $1993=$ R. Syme, L'aristocrazia augustea (trad. it.), Milano, 1993.Tansey $2008=$ P. Tansey, Q. Aemilius Lepidus (Barbula?) cos. 21 B.C., in Historia, 57-2, 2008, p. 174-207.

Tansey $2018=$ P. Tansey, Notabilia varia in the Fasti of Alba Fucens, in SCO, 64, 2018, p. 199-269.

Weigel $1974=\mathrm{R}$. Weigel, Lepidus reconsidered, in Acta Classica, 17, 1974, p. 67-73.

Weigel $1985=$ R. Weigel, Augustus' Relations with the Aemilii Lepidi - Persecution and Patronage, in RhMus, 128, 1985, p. 180-191.

Weigel $1992=\mathrm{R}$. Weigel, Lepidus. The tarnished triumvir, London, 1992.

Welch $1995=\mathrm{K}$. Welch, The career of M. Aemilius Lepidus 49-44 B.C., in Hermes, 123, 1995, p. 443-454.

Welch 2012 = K. Welch, Magnus Pius. Sextus Pompeius and the Transformation of the Roman Republic, Swansea, 2012.

Zevi 2016 = F. Zevi, I Fasti di Privernum, in ZPE, 197, 2016, p. 287-309.

Zevi 2017 = F. Zevi, $I$ Fasti di Privernum alla luce della collaborazione con Filippo Càssola, in M. Chiabà, L. Cristante (a cura di), Il sussurro di una brezza leggera. Ricordo di Filippo Càssola, Trieste, 2017, p. 5-18.

Zucca $1996=\mathrm{R}$. Zucca, Inscriptiones latine liberae rei publicae Africae, Sardiniae et Corsicae, in L'Africa romana, XI, Ozieri, 1996, p. 1425-1489. 


\section{Table des matières}

Premier fascicule

TITE-LIVE ET LA ROME ARCHAÏQUE, RÉCIT OU HISTOIRE?

sous la direction de Nicolas L.J. Meunier

Introduction, par Nicolas L.J. MeUnIER $\ldots \ldots \ldots \ldots \ldots \ldots \ldots \ldots \ldots \ldots \ldots \ldots \ldots \ldots \ldots \ldots$

Lucio Giunio Bruto: caratteri antichi del fondatore della repubblica romana, par Attilio

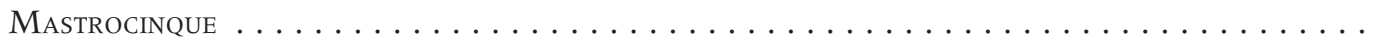

Contaminations culturelles dans le récit livien, par Bernard MinEo

Duels et deuotiones, une représentation de la guerre dans l'historiographie livienne, par Marine

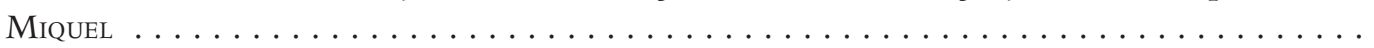

L'organisation politique et territoriale des peuples de l'Italie préromaine vue par Tite-Live, par

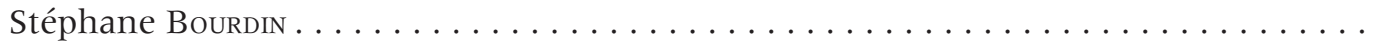

Marcus Manlius Capitolinus entre Rome et le Latium: questions de définition identitaire et de distorsion narrative, par Nicolas L.J. MeUnIER . . . . . . . . . . . . . . . . . . . .

\section{VARIA}

Muro Leccese nell'età del Ferro. Forma e organizzazione insediativa di un abitato indigeno della Puglia meridionale, par Francesco Meo . . . . . . . . . . . . . . . . .

La ricezione del mito di Galatea in Etruria tra antiquaria, epigrafia ed iconografia, par Laura

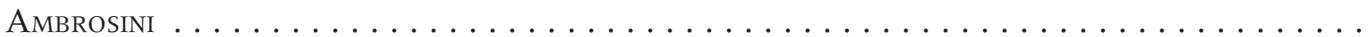

L'onomastica nella ricostruzione del lessico: il caso di Retico ed Etrusco, par Simona MARCHESINI

Le tombe con marchi del sepolcreto di via Sabotino a Bologna, par Dominique Briquel et Luana Kruta Popri . . . . . . . . . . . . . . . . . . . . . . . . . . . . . . .

Rome et la troisième guerre punique: unipolarité méditerranéenne et dilemme de sécurité au II siècle a.C., par Pierre-Luc Brisson . ..............................

CIL IV, 9591 : un transport de blé entre Ostie et Pompéi - II, par Jean ANdreau, Lucia Rossi et André TcherNia . . . . . . . . . . . . . . . . . . . . . . . . . . 
Second fascicule

\section{FAC-SIMILE 1: LE COLLEZIONI DI DOCUMENTAZIONE GRAFICA SULLA PITTURA ETRUSCA}

sous la direction de Lucrezia Cuniglio, Natacha Lubtchansky, Susanna SARTI

Introduzione, par Lucrezia Cuniglio, Natacha Lubtchansky, Susanna Sarti. . . . . . . . . . . .

I. ESPERIENZE MUSEALI A CONFRONTO

Immagini svelate. Le copie al vero di Carlo Ruspi nel Museo Gregoriano Etrusco, par Maurizio

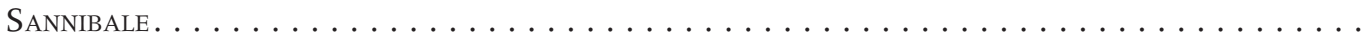

Le riproduzioni di tombe etrusche nella Alte Pinakothek di Monaco attraverso disegni e fotografie,

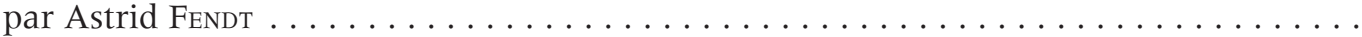

"Ora mi prometterei di fare il primo vero lucido»: Adolfo Cozza e la riproduzione delle tombe Golini I e II, par Giuseppe M. Della Fina . . . . . . . . . . . . . . . . . . . . . .

"Meglio di qualunque descrizione fan conoscere gli usi»: la Galleria delle pitture etrusche del Museo Civico Archeologico di Bologna, par Ornella Chillé, Anna Dore, Marinella Marchesi . . . . . .

From decoration to documentation. The Helbig-Jacobsen facsimiles and their afterlife, par Mette Moltesen ..............................................

Ancora sulle riproduzioni di pitture etrusche a Firenze. Le carte personali di Augusto Guido Gatti (1863-1947), par Lucrezia Cuniglio, Susanna SARTi . . . . . . . . . . . . . . . . . . . . . . .

II. COLLEZIONI DI MATERIALI A STAMPA, DISEGNI E ACQUERELLI

Les dessins étrusques des voyageurs français aux XIX et XXe siècles, par Annie Jacques . ......

Benjamin Schlick: nouveaux dessins étrusques de Corneto (Tarquinia), par Natacha LuBTcHANsKY

Da Carlo Ruspi a Gregorio Mariani. La documentazione grafica della pittura etrusca nell'Archivio dell'Istituto Archeologico Germanico di Roma, par Valeria CAPoBIANCo, Marina Unger . . . . . .

La Tomba François di Vulci: dalla documentazione grafica del Fondo des Vergers della Biblioteca Gambalunga alle decorazioni pittoriche della biblioteca di Villa des Vergers, par Paola DeLBIANCO

Pittura funeraria etrusca nelle copie dell'Istituto Svedese di Roma: la collezione Morani e gli acquerelli Boberg, par Astrid CAPOFERRO . ...........................

Le copie ad acquerello delle pitture funerarie etrusche nel Museo delle Antichità Etrusche e Italiche della Sapienza Università di Roma, par Claudia CARLucci, Laura M. Michettr. . . . . . . . . . . . . 
VARIA

Les sphinx sur le Vase François et l'Olpè Chigi: l'héroïsation des élites, par Thierry PeтrT .....

$431-455$

Abakainon (Sicilia): nuove considerazioni sul paesaggio funerario «monumentale» della necropoli di età tardo-classica ed ellenistica in contrada Cardusa, par Girolamo Sofia . . . . . . . . . . . . .

$457-478$

Su alcuni depositi rituali di Agrigento: prassi sacrificale e «riti di costruzione» in ambito domestico nel Quartiere ellenistico-romano (Insula III, Casa M), par Francesco Belfiori . . . . . . . . . .

Le ultime acquisizioni dal teatro di Terracina e l'eccezionale iscrizione del triumviro M. Emilio Lepido, par Nicoletta Cassieri, Gian Luca Gregori, Jean-Baptiste Refalo-Bistagne . . . . . . .

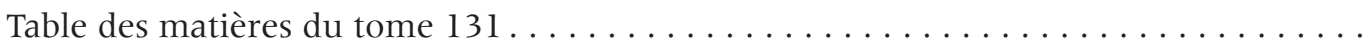


Achevé d'imprimer en juillet 2020 sur les presses de The Factory srl Via Tiburtina, 912 00156 Roma 